\title{
Der Datensatz «Walderschliessungsstrassen 2013» des Schweizerischen Landesforstinventars
}

\author{
Kalin Müller \\ Marielle Fraefel \\ Fabrizio Cioldi \\ Paolo Camin \\ Christoph Fischer
}

Eidgenössische Forschungsanstalt für Wald, Schnee und Landschaft, Forschungsgruppe GIS (CH) Eidgenössische Forschungsanstalt für Wald, Schnee und Landschaft, Forschungsgruppe GIS (CH)* Eidgenössische Forschungsanstalt für Wald, Schnee und Landschaft, Forschungsgruppe Wissenschaftlicher Dienst LFI (CH)

Bundesamt für Umwelt, Abteilung Wald $(\mathrm{CH})$

Eidgenössische Forschungsanstalt für Wald, Schnee und Landschaft, Forschungsgruppe Wissenschaftlicher Dienst LFI $(\mathrm{CH})$

\section{Der Datensatz «Walderschliessungsstrassen 2013» des Schweizerischen Landes- forstinventars}

\begin{abstract}
Informationen über die Erschliessung des Waldes bilden eine bedeutende Grundlage für die Waldbewirtschaftung. Sie dienen beispielsweise dazu, das Holznutzungspotenzial abzuschätzen oder Gebiete mit unzureichender Erschliessung zu ermitteln. Im Rahmen des Schweizerischen Landesforstinventars (LFI) wurde in den Jahren 2013/2014 der Geodatensatz der Walderschliessungsstrassen landesweit auf den neuesten Stand gebracht. Damit liegt eine wertvolle Datengrundlage vor, die flächendeckend die holzabfuhrrelevanten, mit Lastwagen befahrbaren Strassen im Wald beinhaltet. Die Daten wurden mithilfe einer Umfrage bei den rund 800 Revierförstern mittels Kartierung auf Papier und anschliessender Digitalisierung erhoben. Dabei wurden einerseits die Informationen zu den Waldstrassen der LFI-Zeitreihe aktualisiert, andererseits wurden zusätzliche Merkmale bezüglich Befahrbarkeit praxisnah anhand von Lastwagenkategorien erfasst. Die Informationen sind den Geometrien der Strassen des topografischen Landschaftsmodells des Bundesamts für Landestopografie swisstopo (swissTLM 3D, Ausgabe 2012) hinzugefügt. Die Daten eignen sich für statistische Analysen und für Modellierungen; für ortsgenaue Verwendungen, zum Beispiel für ein Navigationssystem, müsste der Datensatz weiter bearbeitet werden. Die Daten werden an der Eidgenössischen Forschungsanstalt für Wald, Schnee und Landschaft (WSL) gepflegt und sind bei nicht kommerzieller Nutzung auch für Dritte erhältlich, sofern eine TLM-Lizenz vorliegt. Im Artikel werden der Datensatz, seine Erhebung und mögliche Anwendungen beschrieben.
\end{abstract}

Keywords: forest transportation system, forest access roads, trafficability, trucks, timber harvesting doi: $10.3188 /$ szf. 2016.0136

*Zürcherstrasse 111, CH-8903 Birmensdorf, E-Mail marielle.fraefel@wsl.ch

$\mathrm{D}$ ie Walderschliessung mit lastwagenbefahrbaren Strassen ist für unterschiedliche Nutzungen des Waldes von Bedeutung, so für die Waldpflege (Schärer 2004), für die Erholungsnutzung (Janowsky \& Becker 2003) oder für den Katastrophenschutz (z.B. bei Waldbränden). Eine besondere Rolle spielt sie für die Nutzung der Ressource Holz: Die Strassen gewährleisten den Abtransport des Holzes und sind damit ein elementares Glied in der Logistikkette von Forst und Holz (Nüsslein 2007, Blattert \& Lemm 2011, Bont et al 2015).

Flächendeckende Informationen zur Erschliessung des Waldes werden in der Schweiz hauptsächlich im Rahmen des Landesforstinventars (LFI) erhoben. Das LFI ist ein langfristig angelegtes Monitoringprojekt, mit dem seit mehr als 30 Jahren der Zustand und die Entwicklung des Waldes erfasst werden (Brändli 2010). Es wird von der Eidgenössischen
Forschungsanstalt für Wald, Schnee und Landschaft (WSL) in Zusammenarbeit mit dem Bundesamt für Umwelt (BAFU) durchgeführt. Zurzeit ist die vierte Aufnahme im Gang (LFI 2009-2017); dabei wird auch die Erschliessungserhebung weitergeführt.

In den letzten Jahrzehnten sind die Fahrzeuge für den Holzabtransport immer grösser geworden. Die bestehenden Walderschliessungsstrassen können mit diesen teilweise nicht mehr befahren werden. Für einen effizienten Holztransport ist es aber wichtig, dass das Erschliessungssystem an die aktuellen Holzernte- und Holztransportsysteme angepasst ist (Enache \& Stampfer 2015). Um beurteilen zu können, inwiefern die heutige Strassenerschliessung den technischen Anforderungen moderner Transportsysteme genügt, wurden in der aktuellen Erschliessungserhebung neben den in den früheren Inventuren erfassten Daten zusätzliche Informatio- 
nen, insbesondere zur Tragfähigkeit und Fahrbahnbreite von lastwagenbefahrbaren Waldstrassen, erhoben. Damitwerden auch Entscheidungsgrundlagen für die Waldpolitik 2020 bereitgestellt; mit dieser will der Bund günstige Rahmenbedingungen für eine optimale Waldbewirtschaftung schaffen und sicherstellen, dass der Wald seine vielfältigen Funktionen erfüllen kann (BAFU 2013).

In diesem Artikel wird der Datensatz «Walderschliessungsstrassen 2013», wie er im Rahmen des vierten LFI in den Jahren 2013/2014 erhoben wurde, vorgestellt, und es werden bereits realisierte und weitere mögliche Anwendungen präsentiert.

\section{Inhalt des Datensatzes}

Seit der ersten Erschliessungserhebung (1983/ 1985) werden im LFI alle Waldstrassen erfasst, die eine Fahrbahnbreite von mindestens $2.5 \mathrm{~m}$ und einen Oberbau, der für Lastwagen mit einer Achslast von 10 t dimensioniert ist, aufweisen (Keller 2013). Strassen ausserhalb des Waldes werden nur in Ausnahmefällen aufgenommen. Die Erfassung nach dieser Definition wurde auch in der aktuellen Erhebung der Jahre 2013/2014 weitergeführt, um Veränderungen zwischen den Inventuren verfolgen und quantifizieren zu können. Zusätzlich zu dieser Zeitreihe wurden im Rahmen einer Zusatzerhebung für alle Walderschliessungsstrassen, die mindestens $3 \mathrm{~m}$ breit und für ein 3-achsiges Fahrzeug (26 t) befahrbar sind, detailliertere Informationen zum Ausbau und zur Dimensionierung aufgenommen (Tabelle 1).

\section{Attribute der LFI-Zeitreihe}

$\mathrm{Zu}$ jeder Strasse beziehungsweise $\mathrm{zu}$ jedem Strassenabschnitt, der die Mindestkriterien der LFIZeitreihe erfüllt, werden Informationen zu den Änderungen im Vergleich zur Vorinventur erhoben, und zwar in den Kategorien «Belagsänderung», «Neu- bau», «Ausbau», «Rückbau», «Sanierungsfall» und «Aufgelassen» (nicht mehr unterhalten). Daneben gibt es Strassen, deren Einteilung korrigiert werden muss, d.h., sie haben keine reale Änderung erfahren, sondern waren in der Vorinventur nicht oder nicht korrekt (Nachführung) beziehungsweise fälschlicherweise erfasst worden (Fehlklassierung). Unter dem Stichwort «Belag» werden drei Arten von Deckschichten unterschieden (vgl. Kuonen 1983): Naturstrasse (wassergebundene Verschleissschicht), Asphalt (bituminöse Bindemittel) und Beton (hydraulische Bindemittel). All diese Informationen werden mit dem Attribut «Linientyp» beschrieben (Tabelle 2). In den Attributen «Verlauf» und «Belag» werden einige der genannten Kategorien zusammengefasst. Der aktuelle Stand der Erschliessung lässt sich somit durch Auswahl der Strassen anhand dieser Attribute darstellen.

\section{Attribute der Zusatzerhebung}

Die zusätzlichen, detaillierteren Eigenschaften wurden nur zu denjenigen Waldstrassen erhoben, die mindestens $3 \mathrm{~m}$ breit und mit einem 3-Achser mit einem Gesamtgewicht von $26 \mathrm{t}$ befahrbar sind. Innerhalb des Waldes wurden alle Strassen erfasst, die der Definition entsprechen und die a) ganzjährig befahrbar sind (bis auf 1-2 Monate) sowie b) für das Rücken oder Lagern von Holz (im Wald und am Waldrand) genutzt werden können. Ausserhalb des Waldes wurden nur die wichtigsten Abfuhrrouten bis zum übergeordneten, jederzeit befahrbaren Strassennetz (z.B. Kantonsstrassen) berücksichtigt. Damit bilden die sich innerhalb des Waldes befindlichen Strassen der Zusatzerhebung eine Teilmenge der Waldstrassen der LFI-Zeitreihe.

Im Datensatz wird die Strassenbreite mit dem Attribut «Breite» (3.0-3.49 m bzw. $\geq 3.5 \mathrm{~m})$ und die grösste erlaubte Lastwagenkategorie (Abbildung 1) mit dem Attribut «Befahrbarkeit» beschrieben (Tabelle 3). Die zu unterscheidenden Kategorien bei diesen zwei Attributen wurden in Zusammenarbeit mit

\begin{tabular}{|c|c|c|c|c|}
\hline Merkmal & \multicolumn{2}{|l|}{ Zeitreihe } & \multicolumn{2}{|l|}{ Zusatzerhebung } \\
\hline Mindestbreite & \multicolumn{2}{|l|}{$2.5 \mathrm{~m}$} & \multicolumn{2}{|l|}{$3.0 \mathrm{~m}$} \\
\hline $\begin{array}{l}\text { Mindestens } \\
\text { befahrbar für }\end{array}$ & \multicolumn{2}{|c|}{ Fahrzeug mit 10 t Achslast } & \multicolumn{2}{|c|}{ 3-Achser mit Gesamtgewicht 26 t } \\
\hline \multirow{6}{*}{$\begin{array}{l}\text { Unterschiedene } \\
\text { Hauptkategorien }\end{array}$} & \multirow[t]{3}{*}{ Belagstyp } & Naturstrasse & \multirow[t]{2}{*}{ Strassenbreite } & $3.0-3.49 \mathrm{~m}$ \\
\hline & & Asphaltstrasse & & $\geq 3.5 \mathrm{~m}$ \\
\hline & & Betonstrasse & \multirow{3}{*}{$\begin{array}{l}\text { Fahrzeugtyp / } \\
\text { Gesamtgewicht }\end{array}$} & 3-Achser / 26 t \\
\hline & \multirow[t]{2}{*}{ Art der Änderung } & \multirow[t]{2}{*}{ Belagsänderung usw. } & & 4-Achser / 28/32t \\
\hline & & & & 5-Achser / 40/44 t \\
\hline & \multicolumn{2}{|l|}{ siehe Tabelle 2} & \multicolumn{2}{|l|}{ siehe Tabelle 3} \\
\hline $\begin{array}{l}\text { Berücksichtigtes } \\
\text { Gebiet }\end{array}$ & \multicolumn{2}{|c|}{ Wald und Waldrand } & \multicolumn{2}{|c|}{$\begin{array}{l}\text { Wald, Waldrand sowie wichtigste Abfuhrrouten } \\
\text { ausserhalb des Waldes }\end{array}$} \\
\hline
\end{tabular}

Tab 1 Gegenüberstellung der Mindestkriterien, der unterschiedenen Kategorien und der berücksichtigten Gebiete für Walderschliessungsstrassen gemäss LFI-Zeitreihe (seit der ersten Erhebung in den Jahren 1983-1985 gültige Definition) und gemäss Zusatzerhebung (erstmalige Aufnahme in den Jahren 2013-2014) im Datensatz «Walderschliessungsstrassen 2013». 
dem BAFU festgelegt und in einer Pilotstudie auf ihre Anwendbarkeit geprüft. Das Attribut «Hindernis» erlaubt es, Strecken mit reduzierter Befahrbarkeit zu markieren. Dabei werden die zwei Typen «bauliches Hindernis» (z.B. Unterführung) und «behördliche Einschränkung» (z.B. Bewilligungspflicht) unter-

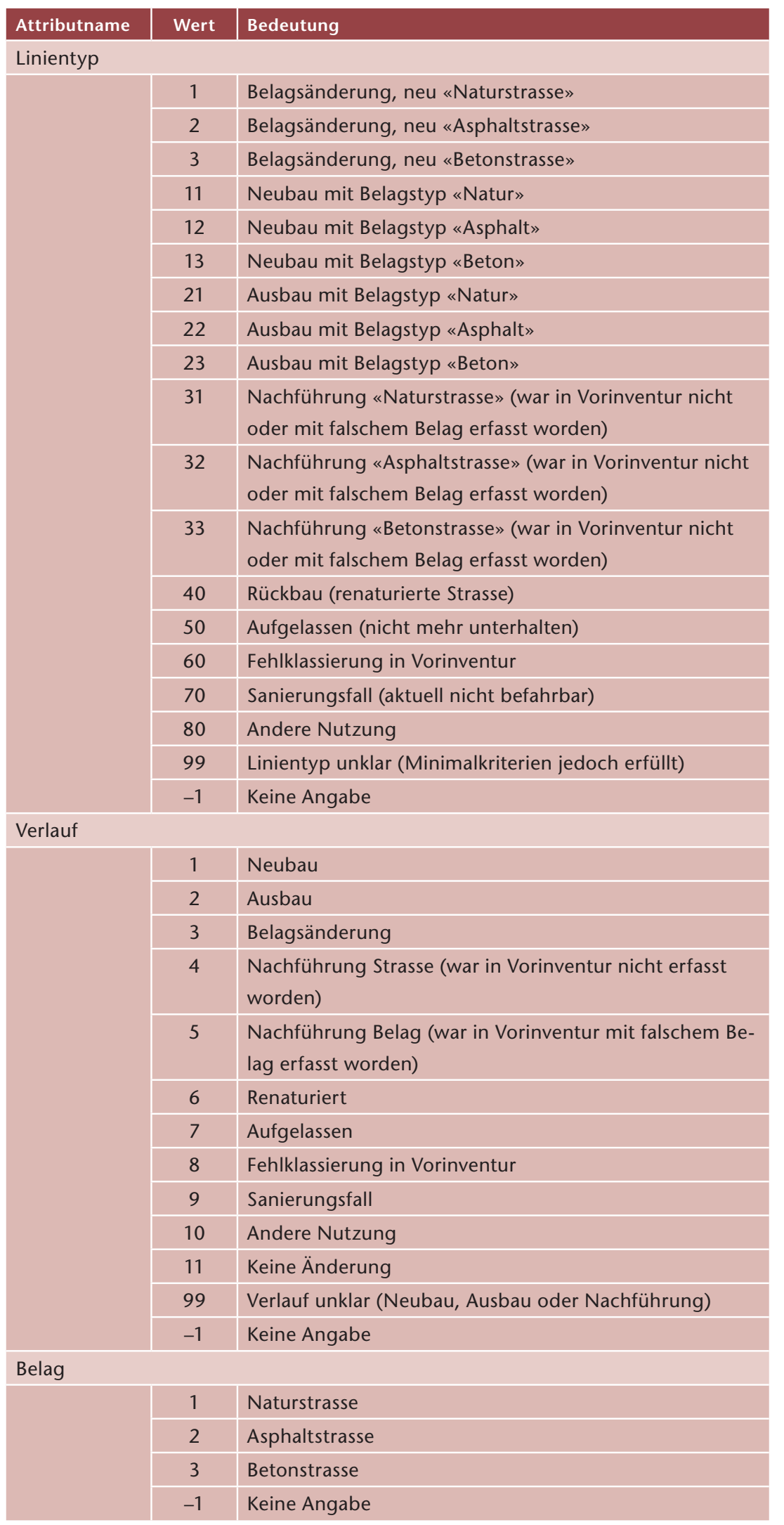

Tab 2 Für die LFI-Zeitreihe erfasste Attribute der Erschliessungsstrassen. Strassen, die die Kriterien der LFI-Zeitreihe nicht erfüllen und entsprechend nicht kartiert wurden, werden mit «-1 (Keine Angabe)» bezeichnet. schieden. Schliesslich wird mittels des Attributs «Huckepack» signalisiert, dass mit einem 3- oder 4-Achser in eine Sackgasse hineingefahren und nach Abladen des Anhängers mit einem 6-Achser herausgefahren werden kann.

\section{Datenerhebung}

\section{Ausgangsdaten}

Bei der dritten Erschliessungserhebung (20042006) wurden die erhobenen Informationen als Attribute an den Vector25-Strassendatensatz der swisstopo angehängt. Im Zuge der Vorbereitungen zur Erhebung 2013-2014 wurden diese Attribute auf die neuen Strassengeometrien des topografischen Landschaftsmodells der swisstopo (swissTLM ${ }^{3 \mathrm{D}}$, Ausgabe 2012) übertragen, sodass auch die Attribute und topologischen Eigenschaften (inkl. Netzwerkeigenschaften) des swissTLM ${ }^{3 \mathrm{D}}$ genutzt werden können. Dieser Datensatz bildet die Grundlage für die Erfassung der aktuellen Situation.

\section{Methodenentwicklung und Pilotinventur}

Als Vorbereitung für die aktuelle Erschliessungserhebung wurde 2012 eine Pilotstudie durchgeführt, um sowohl die Kriterien für die Zusatzerhebung als auch den Ablauf der erweiterten Erhebung zu optimieren. Dafür wurde in sechs Forstrevieren - drei im Kanton Zürich, drei im Kanton Graubünden - die Erfassung der Waldstrassen nach der LFIZeitreihe und gemäss Zusatzerhebung getestet. Diese Tests führten zu Anpassungen bei den Attributen der Zusatzerhebung und am Vorgehen; so wurden die Befahrbarkeits- und Fahrbahnbreitenklassen angepasst und die Darstellung der Strassen auf der Karte in Bezug auf die Farbgebung optimiert. Auch die direkte digitale Erhebung - vor Ort am Computer - im Vergleich zur Erhebung auf einer Papierkarte mit späterer Digitalisierung wurde geprüft. Dabei überwogen die Vorteile der Variante «Papierkarte»: Eine grosse Papierkarte im Massstab 1: 25000 (A1-Format) bietet einen besseren Überblick als die Darstellung auf einem (auch grossen) Bildschirm. Ausserdem erwies sich die direkte digitale Erfassung als so zeitaufwendig für Förster und Interviewer, dass sie im Hinblick auf die Durchführbarkeit des Projekts verworfen werden musste. Stattdessen wurde die Digitalisierung von spezialisierten Mitarbeitern an der WSL durchgeführt.

\section{Befragung}

Die Erschliessungserhebung ist ein Teil der LFI-Umfrage, die bei den rund 800 Revierförstern der Schweiz durchgeführt wird und bei der auch Informationen zu den terrestrischen Waldstichproben erhoben werden (z.B. Waldfunktionen, Planungsgrundlagen, Eigentumsverhältnisse). Insge- 
Abb 1 Verwendete Lastwagenkategorien für die Zusatzerhebung (verändert nach Heinimann 2012).

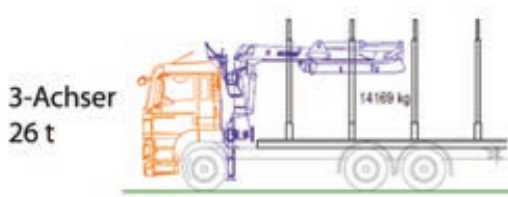

5- bis 6-Achser $40 \mathrm{t} / 44 \mathrm{t}$

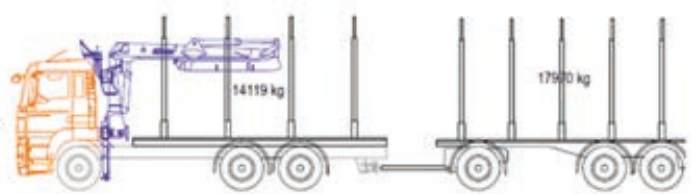

4-Achser $28 \mathrm{t} / 32 \mathrm{t}$

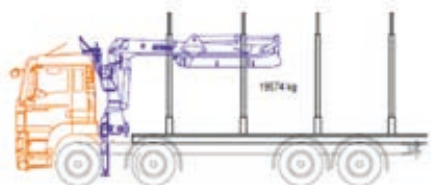

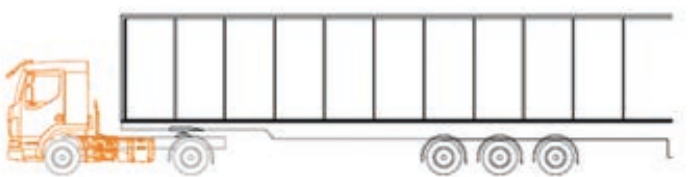

samt neun speziell geschulte LFI-Mitarbeitende führten innert zehn Monaten die rund 800 Interviews anhand der Umfrageanleitung (Keller 2013) durch, die die zu erhebenden Werte und das korrekte Vorgehen bei der Befragung und der Kartierung im Detail beschreibt.

Für die Erhebung der Walderschliessung wurden analoge Karten im Massstab 1:25000 im Format A1 ausgedruckt. Darauf waren die Strassen gemäss dem Erschliessungsstand 2004-2006 dargestellt, mit unterschiedlichen Signaturen für jeden Belagstyp. Zur Orientierung wurden als Hintergrund die Landeskarten 1:25000 von swisstopo verwendet (Abbildung 2).

Während der Befragung wurde das vorgegebene Strassennetz überprüft, allenfalls korrigiert und ergänzt. In einem ersten Schritt waren die Kriterien der LFI-Zeitreihe entscheidend; dabei wurden

\begin{tabular}{|c|c|c|}
\hline Attributname & Wert & Bedeutung \\
\hline \multicolumn{3}{|l|}{ Befahrbarkeit } \\
\hline & 1 & 3-achsiger Lastwagen mit $26 \mathrm{t}$ Gesamtgewicht \\
\hline & 2 & 4-achsiger Lastwagen mit 28 oder 32 t Gesamtgewicht \\
\hline & 3 & $\begin{array}{l}\text { 5- oder 6-achsiger Lastwagen mit } 40 \text { oder } 44 \mathrm{t} \\
\text { Gesamtgewicht }\end{array}$ \\
\hline & -1 & Keine Angabe \\
\hline \multicolumn{3}{|l|}{ Breite } \\
\hline & 1 & $3-3.49 \mathrm{~m}$ \\
\hline & 2 & $\geq 3.5 \mathrm{~m}$ \\
\hline & -1 & Keine Angabe \\
\hline \multicolumn{3}{|l|}{ Hindernis } \\
\hline & 1 & $\begin{array}{l}\text { Hindernistyp H1 (bauliches Hindernis, d.h. unterdimensio- } \\
\text { niert für 3-Achser) }\end{array}$ \\
\hline & 2 & $\begin{array}{l}\text { Hindernistyp H2 (behördliche Einschränkung, z.B. } \\
\text { Schranke) }\end{array}$ \\
\hline & 3 & Hindernis ohne weitere Angabe (baulich oder behördlich) \\
\hline & -1 & Keine Angabe \\
\hline \multicolumn{3}{|l|}{ Huckepack } \\
\hline & 1 & Befahren von Sackgassen mit Huckepack-System \\
\hline & -1 & Keine Angabe \\
\hline
\end{tabular}

Tab 3 In der Zusatzerhebung erfasste Attribute der Erschliessungsstrassen. Mit «-1 (Keine Angabe)» sind die Strassen bezeichnet, die die Kriterien der Zusatzerhebung nicht erfüllen und entsprechend nicht kartiert wurden. zu ergänzende Strassen eingezeichnet und die Werte des Attributs «Linientyp» von Hand auf der Karte eingetragen. In einem zweiten Schritt wurden die Definitionen der Zusatzerhebung berücksichtigt. Hierfür wurden die Strassen mit gut unterscheidbaren alphanumerischen Codes versehen, bestehend aus der Lastwagen- und der Fahrbahnbreitenkategorie (Abbildungen 1 und 2) sowie, falls angebracht, einem Code für Hindernisse oder Huckepack-System (z.B. «A4+» für eine 3 bis $3.49 \mathrm{~m}$ breite Strasse, die für einen 3-Achser mit 26 Tonnen im HuckepackSystem befahrbar ist).

Kantonale Erschliessungsdatensätze wurden während der Umfrage mit dem Forstdienst als Hilfe genutzt, konnten jedoch nicht direkt in den LFI-Datensatz übernommen werden. Dies insbesondere deshalb, weil in den Kantonen unterschiedliche Definitionen für Waldstrassen Anwendung finden.

\section{Aufwand}

Der Zeitbedarf für die gesamte LFI-Umfrage im Rahmen des vierten LFI betrug 4250 Stunden, davon entfielen 3250 Stunden auf die Erschliessungserhebung. Dies entspricht pro Forstrevier durchschnittlich 5.4 beziehungsweise 4.1 Stunden. In diesem Zeitaufwand inbegriffen sind die Ausbildung der Feldleute, die Organisation der Interviews (Terminvereinbarung und Vorbereitungsarbeiten), die Fahrzeit, die Datenerhebung beim Förster (2.7 Stunden pro Forstrevier, davon 1.4 Stunden für die Erschliessungserhebung) sowie die Nachbearbeitung und Abschlussarbeiten. Der Aufwand für die Entwicklung der Methoden, die Digitalisierung (inkl. Ausbildung und Betreuung) und die Qualitätskontrolle ist hier nicht eingeschlossen.

\section{Digitalisierung}

Die rund 500 Karten aus den Umfragen wurden gescannt und georeferenziert. So konnten sie als Hintergrundebene in einem geografischen Informationssystem (GIS) verwendet und die kartierten Angaben in der Form von neuen Attributen auf den digitalen Strassendatensatz übertragen werden. Anschliessend wurde die Konsistenz der Daten überprüft, und Unstimmigkeiten wurden bereinigt. Die 
Konzepte für Kartierung und Digitalisierung waren zusammen entwickelt worden, sodass bei der Digitalisierung mit eindeutigen Angaben gearbeitet werden konnte. Die Digitalisierung erfolgte an der WSL. Die Digitalisierenden und die Projektverantwortlichen für GIS sowie die Projektleitenden für die Datenerhebung und -auswertung arbeiteten eng zusammen. Dies war insbesondere bei Widersprüchen und nicht vorgesehenen Fällen in der Kartierung wichtig. Eigens programmierte Digitalisierungshilfen ermöglichten effiziente Arbeitsabläufe.

\section{Genauigkeit und Vollständigkeit}

Die geometrische Genauigkeit entspricht derjenigen der Strassen des swissTLM ${ }^{3 \mathrm{D}}$, Ausgabe 2012, von swisstopo. Die Strassendaten haben eine Lagegenauigkeit von 1-8 m, je nachdem, ob sie in der verwendeten Ausgabe bereits gemäss TLM-Standard vorlagen oder lediglich vom Vorgängermodell (Vector25) übernommen worden waren.
Inhaltlich beruhen die Angaben zu den Strassen auf den Kenntnissen des Försters über das Forstrevier; es wurden keine Erhebungen im Feld durchgeführt. Wenn es bei der Beurteilung der Fahrbahnbreite Schwierigkeiten gab, wurde im Zweifelsfall konservativ beurteilt und die niedrigere Kategorie gewählt.

Innerhalb des Waldes wurden alle Strassen beurteilt. Ausserhalb des Waldes wurden für die Zusatzerhebung nur die wichtigsten Abfuhrrouten aus dem Wald erhoben, was dazu führt, dass es Waldstücke mit Strassen gibt, die nicht an das übergeordnete Strassennetz angebunden sind.

\section{Nutzen für die Praxis und Ausblick}

Im LFI werden die Erschliessungsdaten der LFI-Zeitreihe dafür genutzt, den Zustand und die Veränderung der Walderschliessung in der Schweiz

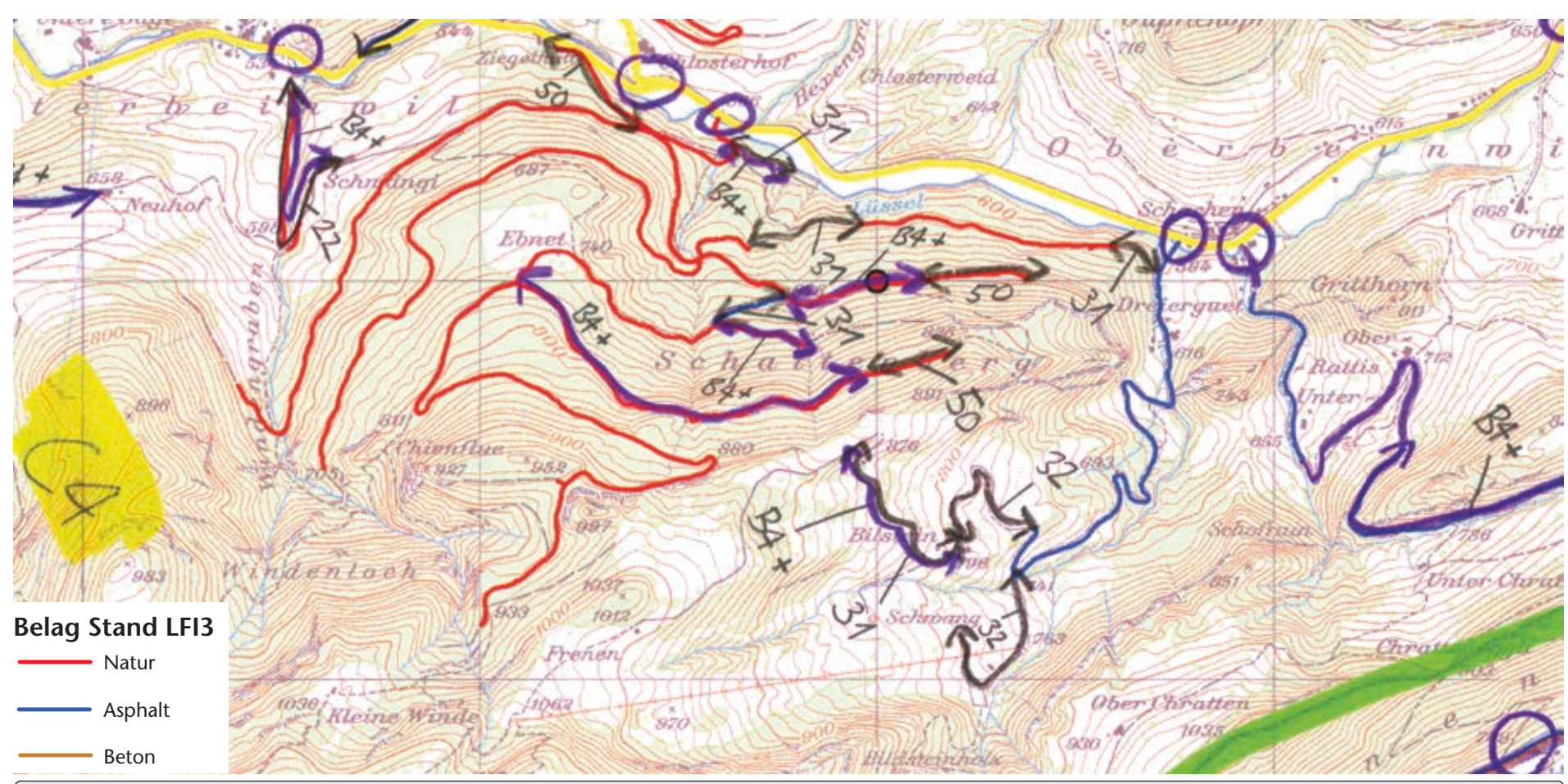

\begin{tabular}{|c|c|c|c|c|c|c|}
\hline \multirow{10}{*}{ Signaturen für Änderungen } & \multicolumn{6}{|l|}{ Code zu Linientyp LFI4 } \\
\hline & \multirow{3}{*}{ Bisherige LKW-Strasse, nur Belagsänderung } & ohne Belag & 01 & \multirow{3}{*}{ Nachführung (war schon LKW-Strasse in Vorinventur) } & ohne Belag & 31 \\
\hline & & Asphalt & 02 & & Asphalt & 32 \\
\hline & & Beton & 03 & & Beton & 33 \\
\hline & \multirow{3}{*}{ Strassenneubau } & ohne Belag & 11 & & & \\
\hline & & Asphalt & 12 & \multicolumn{2}{|l|}{\begin{tabular}{|l} 
Rückbau (renaturierte Strasse) \\
\end{tabular}} & 40 \\
\hline & & Beton & 13 & \multicolumn{2}{|l|}{\begin{tabular}{|l|l} 
Aufgelassen (kein Unterhalt) \\
\end{tabular}} & 50 \\
\hline & \multirow{3}{*}{ Strassenausbau } & ohne Belag & 21 & \multirow{2}{*}{\multicolumn{2}{|c|}{\begin{tabular}{|l} 
Fehlklassierung (war keine LKW-Strasse in Vorinventur) \\
Sanierungsfall
\end{tabular}}} & 60 \\
\hline & & Asphalt & 22 & & & 70 \\
\hline & & Beton & 23 & \multicolumn{2}{|l|}{\begin{tabular}{|l|} 
Andere Nutzung \\
\end{tabular}} & 80 \\
\hline
\end{tabular}

\section{Zusatzerhebung}

$\stackrel{\text { B4 }}{\longrightarrow}$ Befahrbarkeit/Breite (Beispiel-Code)

+ Hindernis

$\stackrel{\text { A4+ }}{\longrightarrow}$ Huckepack: rein mit A (oder B), raus mit C
Code zu Befahrbarkeit / Breite

\begin{tabular}{|l|l|l|}
\hline Befahrbarkeit & 3-achsiger LKW mit 26t & A \\
\cline { 2 - 3 } & 4-achsiger LKW mit 28t oder 32t & B \\
\cline { 2 - 3 } & min. 5-achsiger LKW mit 40t oder 44t & C \\
\hline
\end{tabular}

\begin{tabular}{|l|l|l|}
\hline \multirow{2}{*}{ Fahrbahnbreite } & $3.00-3.49 \mathrm{~m}$ & 4 \\
\cline { 2 - 3 } & $\sim 3.50 \mathrm{~m}$ & 5 \\
\hline
\end{tabular}
Code zu Hindernis

\begin{tabular}{|l|l|l|}
\hline \multirow{2}{*}{ Hindernis } & Hindernis & $\mathrm{H} 1$ \\
\cline { 2 - 3 } & Behördliche Einschränkung & $\mathrm{H} 2$ \\
\hline
\end{tabular}

Huckepacksystem bei Sackgassen

Abtransport mit C

Abb 2 Ausschnitt einer Kartierung im Kanton Solothurn. Die alphanumerischen Codes wurden bei der Digitalisierung in numerische Werte umgewandelt (Tabellen 2 und 3). 
auszuweisen. Anhand der Erschliessungsdichte (Strassenlänge pro Hektare Wald) und der Distanz von einer Stichprobenfläche bis zur nächsten Waldstrasse können regional differenzierte Aussagen über die Güte der Erschliessung gemacht werden (Brändli et al 2016, dieses Heft). Die Angaben können anhand des Attributs «Belag» weiter differenziert werden. Damit kann beispielsweise ausgesagt werden, dass schweizweit Naturstrassen mit wassergebundener Verschleissschicht bei Waldstrassen am häufigsten sind, auf der Alpensüdseite jedoch solche mit Asphaltbelag dominieren (Brändli 2010). In Kombination mit Merkmalen aus der Felderhebung können weitergehende Analysen gemacht werden. So lässt die Kombination mit dem Holzvorrat Aussagen über das Holzerntepotenzial zu; in Kombination mit dem Gelände liefert der Strassendatensatz Grundlagen für Erschliessungskonzepte oder die Beurteilung von Holzernteverfahren (Brändli 2010). Der Erschliessungsdatensatz der Erhebung 2004-2006 wurde auch für Netzwerkanalysen in Holztransportmodellen verwendet (Steubing et al 2014).

Die differenzierte Betrachtung der Erschliessungssituation nach Lastwagenkategorie erlaubt eine genauere Beurteilung der Holzerntepotenziale. Damit unterstützt der Datensatz die Umsetzung der Schweizer Waldpolitik 2020 (BAFU 2013) und dient dem BAFU zur Steuerung seiner Aktivitäten. Der Nutzen des Datensatzes für Kantone oder Forstbetriebe liegt unter anderem in der Kombination mit selbst erhobenen Informationen. So hat beispielsweise der Kanton Zürich die Erschliessungsinformation des LFI-Datensatzes 2004-2006 mit seinen eigenen Strasseninformationen zusammengeführt, der Kanton Basel-Landschaft hat den Datensatz für die Optimierung seines Rückegassennetzes verwendet, und im Kanton Freiburg wurden die Waldinfrastrukturen im kantonalen GIS ergänzt.

Allgemein eignet sich der Datensatz «Walderschliessungsstrassen 2013» gut für statistische Analysen innerhalb des Waldes, wo die Strassen vollständig erhoben wurden. Weil mit der Zusatzerhebung auch die wichtigsten Abfuhrrouten ausserhalb des Waldes bis zum übergeordneten Strassennetz erfasst wurden, kann der Datensatz auch für Netzwerkanalysen verwendet werden; dies ermöglicht zahlreiche interessante Untersuchungen, von der Abschätzung von Holzerntekosten bis hin zur konkreten Walderschliessungsplanung. Die Durchführung solcher Netzwerkmodellierungen ist im LFI bereits geplant. Weiter wird zurzeit abgeklärt, wie flächendeckend ein zusammenhängendes Netzwerk für alle Waldstücke erreicht werden kann - auch für diejenigen, für die bisher keine «wichtige» Abfuhrroute kartiert wurde, die aber möglicherweise trotzdem an das übergeordnete Strassennetz angebunden sind.

Auch die Erstellung eines Navigationssystems für die Holzernte auf der Grundlage des Datensatzes
«Walderschliessungsstrassen 2013» wäre denkbar. Analog zum Waldnavigationssystem von NavLog $\mathrm{GmbH}^{1}$ in Deutschland könnte das übergeordnete Strassennetz aus herkömmlichen Navigationssystemen mit dem Datensatz des LFI kombiniert werden (Blattert \& Lemm 2011). Dazu müsste der Datensatz allerdings weiter bearbeitet und stets auf aktuellem Stand gehalten werden. Dies würde die Kapazitäten des LFI übersteigen. Ein möglicher Ansatz dazu wäre, dass der jetzige Datensatz von den Kantonen - beispielsweise mittels Web-Editing - weitergepflegt würde.

\section{Datenabgabe}

Der Datensatz «Walderschliessungsstrassen 2013» (Erhebung 2013-2014) ist auf der Website www.datacite.org registriert und mit dem Digital Object Identifier (doi) 10.16904/3 zitierbar (Kasten 1). Im Rahmen einer vertraglichen Vereinbarung wird der Datensatz - wie andere Daten des LFI - für nicht kommerziellen Gebrauch zur Verfügung gestellt. Je nach Verwendungszweck können die Daten für die gesamte Schweiz oder für Teilgebiete (z.B. einen Kanton) bestellt werden. Abgegeben werden können die in einem GIS gespeicherten Daten im Shapefile-Format oder als Feature Class in einer File Geodatabase. Der Empfänger muss hierfür allerdings über eine Lizenz für Strassen des swissTLM ${ }^{3 D}$ (Ausgabe 2012) von swisstopo verfügen.

Eingereicht: 19. Oktober 2015, akzeptiert (mit Review): 21. März 2016

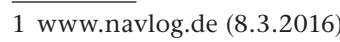

\begin{tabular}{|c|c|}
\hline Identifizierung & doi:10.16904/3 \\
\hline Urheber & Landesforstinventar LFI, www.Ifi.ch \\
\hline Affiliation & $\begin{array}{l}\text { Schweizerische Forschungsanstalt } \\
\text { für Wald, Schnee und Landschaft } \\
\text { (WSL), Zürcherstrasse 111, } \\
\text { CH-8903 Birmensdorf; Bundesamt } \\
\text { für Umwelt (BAFU), CH-3003 Bern }\end{array}$ \\
\hline Titel & Walderschliessungsstrassen 2013 \\
\hline Herausgeber & $\begin{array}{l}\text { Schweizerische Forschungsanstalt } \\
\text { für Wald, Schnee und Landschaft } \\
\text { (WSL), Bundesamt für Umwelt } \\
\text { (BAFU) }\end{array}$ \\
\hline Veröffentlichung & 2016 \\
\hline Typ & Geodatensatz \\
\hline Datenaufnahme & $2013-2014$ \\
\hline Kontakt & Landesforstinventar LFI, www.Ifi.ch \\
\hline
\end{tabular}

Kasten 1 Detailinformationen zum Datensatz. 


\section{Literatur}

BLATTERT C, LEMM R (2011) Ein Navi für die Holzabfuhr. Wald Holz 92 (8): 38-40.

BONT L, HEINIMANN H, CHURCH R (2015) Concurrent optimization of harvesting and road network layouts under steep terrain. Ann Oper Res 232: 41-64.

BRÄNDLI UB, EDITOR (2010) Schweizerisches Landesforstinventar. Ergebnisse der dritten Erhebung 2004-2006. Birmensdorf: Eidgenöss Forsch.anstalt WSL. 312 p.

BRÄNDLI UB, FISCHER C, CAMIN P (2016) Stand der Walderschliessung mit Lastwagenstrassen in der Schweiz. Schweiz Z Forstwes 167: 143-151. doi: 10.3188/szf.2016.0143

BAFU, EDITOR (2013) Waldpolitik 2020. Visionen, Ziele und Massnahmen für eine nachhaltige Bewirtschaftung des Schweizer Waldes. Bern: Bundesamt Umwelt. 66 p.

ENACHE A, STAMPFER K (2015) Machine utilization rates, energy requirements and greenhouse gas emissions of forest road construction and maintenance in Romanian mountain forests. J Green Engin 4: 325-350.

HEINIMANN HR (2012) Unterlagen zur Vorlesung 701-0005-00 an der ETH Zürich «Erschliessungs- und Erntesysteme der Landnutzung». Zürich: Eidgenöss Techn Hochschule.

JANOWSKY D, BECKER G (2003) Characteristics and needs of different user groups in the urban forest of Stuttgart. J Nat Conserv 11: 251-259.
KELLER MR (2013) Schweizerisches Landesforstinventar. Anleitung für die Umfrage- und Erschliessungserhebung 2013. Birmensdorf: Eidgenöss Forsch.anstalt WSL. 40 p.

KUONEN V (1983) Wald- und Güterstrassen. Planung - Projektierung - Bau. Pfaffhausen: Eigenverlag. 743 p.

NÜSSLEIN S (2007) Waldnavigation bringt Holz in Bewegung. Freising: Bayer Landesanstalt Wald Forstwirtschaft, LWF aktuell 56: 6-7.

SCHÄRER W (2004) Der Schutzwald und seine Bedeutung in der Waldpolitik des Bundes. In: WSL, editor. Schutzwald und Naturgefahren. Birmensdorf: Eidgenöss Forsch. Anstalt WSL, Forum für Wissen 2004. pp. 87-90.

STEUBING B, BALLMER I, GASSNER M, GERBER L, PAMPURI L ET $A L$ (2014) Identifying environmentally and economically optimal bioenergy plant sizes and locations: A spatial model of wood-based SNG value chains. Renew Energ 61: 57-68.

\section{Quellen}

Bundesamt für Landestopografie swisstopo. VECTOR25. Das digitale Landschaftsmodell der Schweiz, Stand 2006.

Bundesamt für Landestopografie swisstopo. swissTLM ${ }^{3 D}$ Version 1.1 Ausgabe 2012 - Nachführungsinformation.

Bundesamt für Landestopografie swisstopo. Digitale Landeskarten $1: 25000$ - Pixelkarten, Stand 2013.

\section{Le jeu de données «desserte forestière 2013» de l'Inventaire forestier national suisse}

La connaissance de la desserte est un pilier de la gestion forestière et permet notamment de déterminer le potentiel d'exploitation ou d'identifier les zones difficilement accessibles. Au cours des années 2013-2014, nous avons mené une étude en Suisse afin d'actualiser les données géoréférencées sur la desserte forestière. L'étude s'est déroulée dans le cadre de I'Inventaire forestier national (IFN) et regroupe, à l'échelle nationale, les informations sur les routes forestières praticables par les camions transportant les grumes. L'étude repose sur une enquête auprès des quelque 800 gardes forestiers suisses. Les informations, d'abord reportées sur des cartes topographiques, ont ensuite été numérisées. Cela a permis, d'une part, de mettre à jour les données sur les routes forestières des séries IFN. D'autre part, des paramètres supplémentaires ont été recensés concernant l'accessibilité aux catégories de camions telles qu'utilisées par les praticiens. Les informations recueillies ont été ajoutées aux géométries du réseau de transport de swissTLM3D (version 2012) de l'Office fédéral de topographie swisstopo. Les données se prêtent à des analyses statistiques et des modélisations; pour des applications exigeant une localisation précise comme par exemple les systèmes de navigation, une validation supplémentaire et conséquente est nécessaire. Les données sont conservées à I'Institut fédéral de recherches sur la forêt, la neige et le paysage (WSL) et disponibles à des fins non commerciales pour les tiers pourvu qu'ils possèdent une licence TLM. Cet article décrit le jeu de données, la méthode de son obtention et ses applications possibles.

\section{The Swiss National Forest Inventory dataset «Forest Access Roads 2013»}

Information on forest accessibility is important for forest management. For example, it helps to determine the potential wood supply and identify areas difficult to access. In 2013 2014, a survey was conducted in Switzerland to update the Forest Access Roads geo-dataset within the framework of the Swiss National Forest Inventory (NFI). The resulting nationwide dataset contains valuable information on truck-accessible forest roads that can be used to transport wood. The survey involved interviewing staff from the approximately 800 local forest services in Switzerland and recording the data first on paper maps and then in digitized form. The data in the NFI on the forest roads could thus be updated and additional information regarding their trafficability for specific categories of truck included. The information has now been attached to the geometries of the Roads and Tracks of the swissTLM ${ }^{3 D}$ (release 2012) of the Federal Office of Topography swisstopo. The resulting data are suitable for statistical analyses and modeling, but further (labour-intensive) validation work would be necessary if they are to be used as a basis for applications requiring more spatial accuracy, such as navigation systems. The data are managed at the Swiss Federal Institute for Forest, Snow and Landscape Research (WSL) and are available for third parties for non-commercial use provided they have purchased a TLM license. In this article, the dataset, as well as its acquisition and potential uses, are described. 